\title{
Controlled Introduction of Defects to Delafossite Metals by Electron Irradiation
}

\author{
V. Sunko ${ }^{1,2, *}$ P. H. McGuinness $\odot,{ }^{1,2, \dagger}$ C. S. Chang $\odot,{ }^{3,4}$ E. Zhakina, ${ }^{1,2}$ S. Khim $\odot,{ }^{1}$ C. E. Dreyer, ${ }^{5,6}$ M. Konczykowski $\odot,{ }^{7}$ \\ H. Borrmann, ${ }^{1}$ P. J. W. Moll, ${ }^{1,}$ M. König, ${ }^{1}$ D. A. Muller, ${ }^{3,8}$ and A. P. Mackenzie ${ }^{1,2, \$}$ \\ ${ }^{1}$ Max Planck Institute for Chemical Physics of Solids, Nöthnitzer Straße 40, 01187 Dresden, Germany \\ ${ }^{2}$ SUPA, School of Physics and Astronomy, University of St Andrews, \\ St Andrews KY16 9SS, United Kingdom \\ ${ }^{3}$ School of Applied and Engineering Physics, Cornell University, Ithaca, New York 14853, USA \\ ${ }^{4}$ Department of Physics, Cornell University, Ithaca, New York 14853, USA \\ ${ }^{5}$ Department of Physics and Astronomy, Stony Brook University, Stony Brook, New York 11794-3800, USA \\ ${ }^{6}$ Center for Computational Quantum Physics, Flatiron Institute, \\ 162 5th Avenue, New York, New York 10010, USA \\ ${ }^{7}$ Laboratoire des Solides Irradis, CEA/DRF/IRAMIS, Ecole Polytechnique, \\ CNRS, Institut Polytechnique de Paris, F-91128 Palaiseau, France \\ ${ }^{8}$ Kavli Institute at Cornell for Nanoscale Science, Ithaca, New York 14853, USA
}

(Received 12 December 2019; revised manuscript received 11 March 2020; accepted 18 March 2020; published 24 April 2020)

\begin{abstract}
The delafossite metals $\mathrm{PdCoO}_{2}, \mathrm{PtCoO}_{2}$, and $\mathrm{PdCrO}_{2}$ are among the highest conductivity materials known, with low-temperature mean free paths of tens of microns in the best as-grown single crystals. A key question is whether these very low resistive scattering rates result from strongly suppressed backscattering due to special features of the electronic structure or are a consequence of highly unusual levels of crystalline perfection. We report the results of experiments in which high-energy electron irradiation was used to introduce point disorder to the Pd and Pt layers in which the conduction occurs. We obtain the cross section for formation of Frenkel pairs in absolute units, and cross-check our analysis with first-principles calculations of the relevant atomic displacement energies. We observe an increase of resistivity that is linear in defect density with a slope consistent with scattering in the unitary limit. Our results enable us to deduce that the as-grown crystals contain extremely low levels of in-plane defects of approximately $0.001 \%$. This confirms that crystalline perfection is the most important factor in realizing the long mean free paths and highlights how unusual these delafossite metals are in comparison with the vast majority of other multicomponent oxides and alloys. We discuss the implications of our findings for future materials research.
\end{abstract}

DOI: 10.1103/PhysRevX.10.021018

Subject Areas: Condensed Matter Physics, Materials Science

\section{INTRODUCTION}

Throughout the early evolution of the physics of metals, it was assumed that extremely high metallic state electrical conductivity would be restricted to elemental metals, which could be purified and then annealed to remove dislocations and other structural defects, resulting in long electron mean

\footnotetext{
*Veronika.Sunko@cpfs.mpg.de

†Philippa.McGuinness@cpfs.mpg.de

¥Andy.Mackenzie@cpfs.mpg.de

${ }^{\S}$ Institute of Materials, École Polytechnique Fédérale (EPFL), 1015 Lausanne, Switzerland.

Published by the American Physical Society under the terms of the Creative Commons Attribution 4.0 International license. Further distribution of this work must maintain attribution to the author(s) and the published article's title, journal citation, and DOI. Open access publication funded by the Max Planck Society.
}

free paths. After decades of materials research and refinement, techniques such as heterodoping of semiconductor devices led to the creation of extremely long mean free paths in two-dimensional electron gases (2DEGs), most notably those fabricated at the GaAs/GaAlAs interface. Although painstaking refinement of semiconductor 2DEGs has continued, resulting in exquisite levels of purity [1], the past decade has seen rapid parallel developments in other materials. The insights obtained from the investigations of graphene [2] and of topological insulators [3,4] led to rapid advances in the study of Dirac and Weyl semimetals [5], in which resistivities in the $\mathrm{n} \Omega \mathrm{cm}$ range have been observed [6-8]. These spectacular observations, however, result from materials physics different from that of normal semiconductors. Rather than being materials of particularly high perfection, the main reason for the low resistivity of Weyl and Dirac semimetals is protection from backscattering due to the exotic features of both their bulk band structure and 
the surface states that they host [9]. Strong evidence in favor of this interpretation comes from the ratio of the resistive mean free path to that determined by analysis of de Haas-van Alphen (dHvA) oscillations. The resistive mean free path, which is mainly sensitive to backscattering, is a factor of $\sim 10^{4}$ larger than the dHvA-derived one, which is limited by small-angle scattering events that make only a small contribution to resistivity [6-8].

A further class of materials recently discovered to have extremely long mean free paths at low temperature are the delafossite oxide metals $\mathrm{PdCoO}_{2}, \mathrm{PtCoO}_{2}$, and $\mathrm{PdCrO}_{2}$ $[10,11]$. Each can have resistive mean free paths of microns at low temperatures [12-14]. Indeed, in the most conductive single crystals of $\mathrm{PdCoO}_{2}$, with resistivities as low as $8 \mathrm{n} \Omega \mathrm{cm}$, mean free paths as long as $20 \mu \mathrm{m}$ have been reported [15]. These extremely long mean free paths have led to the observation of a number of novel transport properties [16-21], and there is every prospect of further discoveries. The observation that metallic oxides could have mean free paths as long as this is surprising, especially since they appear in crystals grown using fairly standard methods and not subject to any postgrowth annealing. Put at its simplest, the key question is whether the huge resistive mean free paths are primarily the result of scattering suppression, as in Dirac and Weyl materials, or of unprecedented levels of crystalline perfection. A first clue to the answer to this question came from a study of scattering suppression due to momentum-orbital locking around the Fermi surface of $\mathrm{PtCoO}_{2}$ [22]. Scattering is predicted to be suppressed by a factor of order 2-4 from that in a material with a trivial Fermi surface of the same size. Although this aspect of the physics of the delafossite metals is important, the predicted scattering suppression is nowhere near sufficient to account for the observed lowtemperature mean free paths. Consistent with this finding, the ratio between the resistive and $\mathrm{HHvA}$ mean free paths is a much more modest factor of 10-20 [14,15] than the $10^{4}$ reported in the Dirac and Weyl materials. In combination, the transport data and the analysis presented in Ref. [22] suggest that the delafossite metals might naturally exist with a level of purity that is more or less never observed in as-grown crystals. If that were the case, it would be extremely surprising, and it would open new avenues of research in quantum materials.

In this paper, we report on a combined experimental and theoretical investigation of the disorder dependence of resistivity in $\mathrm{PtCoO}_{2}, \mathrm{PdCoO}_{2}$, and $\mathrm{PdCrO}_{2}$. X-ray diffraction and wide-scale scanning transmission electron microscopy (STEM) confirm the extremely high structural purity of the as-grown single crystals, showing no evidence of layer defects or dislocations over length scales of tens of microns. No point defects such as intersite substitutions or vacancies could be resolved by high-resolution STEM in which atomic resolution has been achieved, but the detection sensitivity for such defects is not sufficient to fully address the issue of ultrahigh purity. To investigate that in depth, we turn to deliberate irradiation. Using 2.5 MeV electrons, we create controlled densities of Frenkel pairs, i.e., displaced atoms leading to a vacancy and an interstitial atom. The kinetics of our experiment are such that no damage cascades are created, and genuine point disorder is achieved. By calculating the absolute cross section for Frenkel pair creation, we prove that the defect scattering is not hugely suppressed in these metals, and show that the point defect density in the as-grown crystals is approximately $0.001 \%$. In other words, the extremely long mean free paths of the delafossite metals are indeed primarily due to a highly unusual level of crystalline perfection. In order to investigate this remarkable fact, and cross-check the analysis that was used to deduce it, we perform first-principles calculations of the atomic displacement energies in delafossites.

The paper is organized as follows. In Sec. II we describe the delafossite crystal structure and sample growth and show transmission electron micrographs and x-ray diffraction patterns of as-grown crystals. We then describe the irradiation experiments and the calculation of the Frenkel pair production cross section in Sec. III, before discussing the dependence of resistivity on defect density in Sec. IV. First-principles calculations are presented in Sec. V, before we close the paper with examples of the changes to transport properties produced by the irradiation in Sec. VI, and a discussion and conclusions in Secs. VII and VIII, respectively.

\section{SAMPLE GROWTH AND IMAGING}

The delafossite crystal structure is shown in Fig. 1(a). It is highly anisotropic, with the Pt or Pd layers separated by layers of cobalt oxide. Each layer is triangularly coordinated, and the interlayer repeat gives the space group $R \overline{3} \mathrm{~m}$ $\left(D_{3 d}^{5}\right)$. Highly two-dimensional conduction $\left(\rho_{c} / \rho_{a b} \sim 10^{3}\right)$ takes place in the $\mathrm{Pd} / \mathrm{Pt}$ layers, while the $\mathrm{CoO}_{2}$ layers can (a)

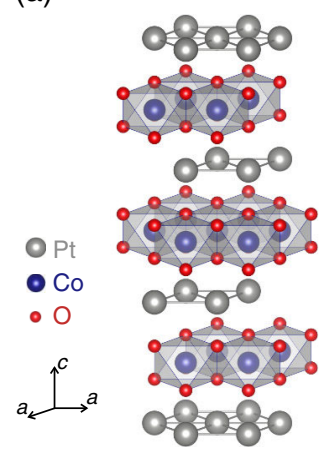

(b)

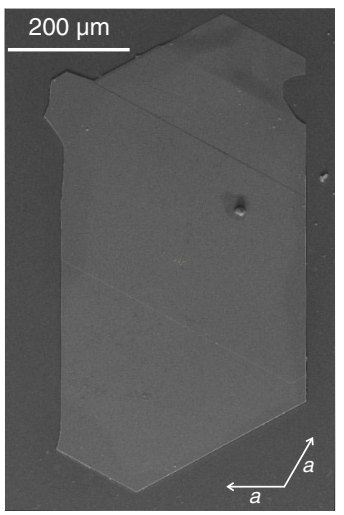

FIG. 1. (a) Delafossite crystal structure, shown on the example of $\mathrm{PtCoO}_{2}$. (b) Scanning electron microscopy (SEM) image of a crystal of $\mathrm{PtCoO}_{2}$. 
(a)

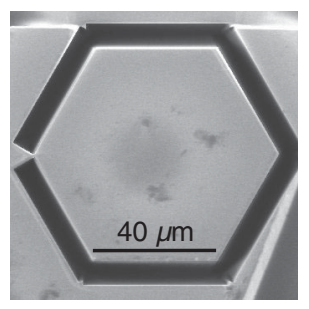

(c)

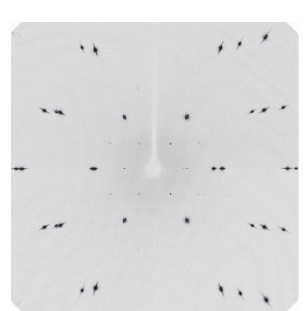

(e)

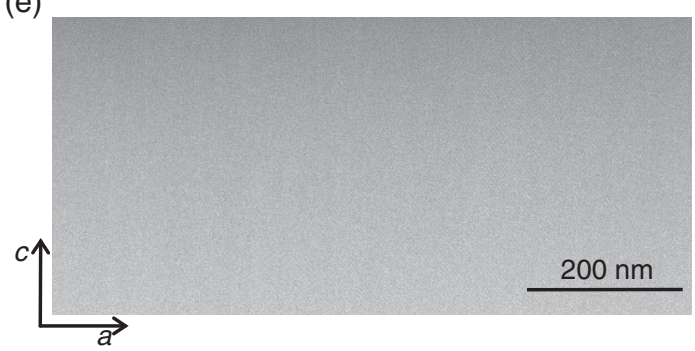

(b)

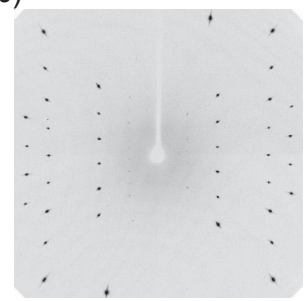

(d)

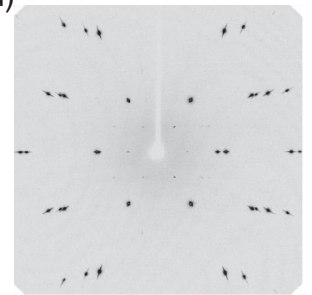

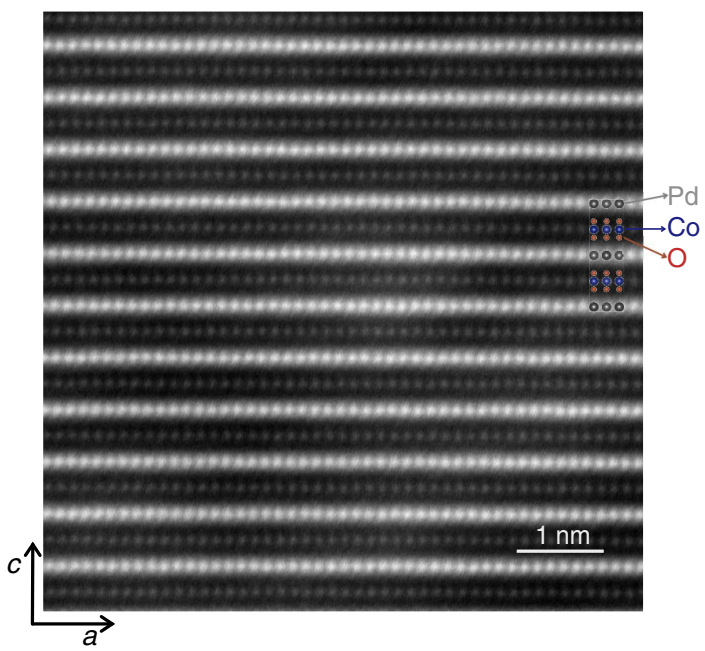

FIG. 3. STEM image of a pristine $\mathrm{PdCoO}_{2}$ sample taken at high magnification resolving $\mathrm{Pd}$ and $\mathrm{Co}$ atomic sites using high-angle annular dark field (HAADF STEM) imaging.

this form of disorder was introduced by the mechanical cleaving usually used to prepare the crystals for diffraction experiments. Preparing the crystals using gentler focused ion beam (FIB) processing [see Fig. 2(a)] resulted in essentially perfect diffraction patterns, with no double peaks that would be a sign of stacking disorder. Example diffraction patterns are shown in Figs. 2(b)-2(d). These images, taken using monochromatic radiation in transmission mode with a beam diameter of $250 \mu \mathrm{m}$, provide true bulk information with high sensitivity towards small distortions or offsets of individual domains. The FIB can also be used, following standard procedures, to thin down samples in order to image them using scanning transmission electron microscopy. In total, we prepared 10 delafossite samples (four $\mathrm{PtCoO}_{2}$ and six $\mathrm{PdCoO}_{2}$ ), allowing us to look at a combined volume of $\sim 45 \mu \mathrm{m}^{3}$. These experiments give results consistent with those from the $\mathrm{x}$-ray diffraction: large-scale defect structures are sometimes seen, but we commonly observed areas of several microns in lateral dimensions over which no defects at all were resolved. A representative image taken on $\mathrm{PtCoO}_{2}$ is shown in Fig. 2(e).

In Fig. 3 we show a high-angle annular dark field STEM image of a $\mathrm{PdCoO}_{2}$ sample, in which atomic contrast has been achieved. No point defects are visible in the image, which is representative of multiple measurements on several samples. This of course does not rule out the existence of point defects, such as interstitials or vacancies, but it does indicate that the concentration of such defects is beyond the resolution of STEM.

\section{ELECTRON IRRADIATION}

Because the defect concentration in as-grown crystals is too low to be directly determined, investigating its influence on resistivity requires deliberate and controlled 


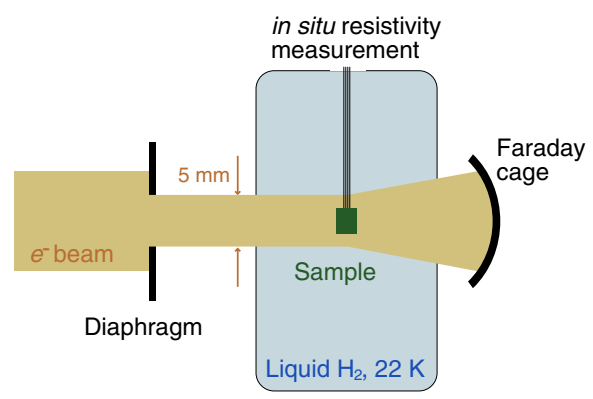

FIG. 4. A sketch of the experimental setup for electron irradiation. The yellow area indicates the range in which the electron beam is swept, rather than representing the beam cross section.

introduction of disorder. Crucially, in order to mimic the situation in the as-grown materials, no large voids or columnar defects should be created, nor should foreign atoms be implanted in the crystal. Instead, individual point defects should be introduced. Irradiation by high-energy electrons in the energy range of $1-10 \mathrm{MeV}$ is the ideal technique to achieve this type of disorder: electrons of these energies can transmit enough energy to an atom to displace it from its lattice site, but not enough for the displaced atom to create a significant number of additional defects. Therefore, individual Frenkel pairs are created. This is in contrast to ion irradiation, where the larger mass of the incoming particle turns every collision into a collision cascade, creating large columnar defects.

\section{A. Experimental setup}

\section{Beam line}

The irradiation with electrons with a maximum kinetic energy of $2.5 \mathrm{MeV}$ was performed at the SIRIUS Pelletron linear accelerator operated by the Laboratoire des Solides Irradiés (LSI) at the Ecole Polytechnique in Palaiseau, France. A sketch of the experimental setup is shown in Fig. 4. During the irradiation the sample was immersed in a bath of liquid hydrogen at a temperature of $\approx 22 \mathrm{~K}$, ensuring that the introduced defects are not mobile. The accelerator beam carried a current in the range of 5-8 $\mu \mathrm{A}$, and passed through a circular diaphragm aperture of a $5 \mathrm{~mm}$ diameter before reaching the sample. The current passing through the diaphragm was experimentally determined using a control metallic sample and found to be in the range of $1.5-2.5 \mu \mathrm{A}$, corresponding to current densities at the sample location of $8-13 \mu \mathrm{A} / \mathrm{cm}^{2}$. The beam was swept vertically and horizontally at two incommensurate frequencies, ensuring the homogeneous irradiation of the sample area. The homogeneous irradiation throughout the sample thickness was guaranteed by the large penetration range of electrons, estimated to be $\sim 1.8 \mathrm{~mm}$ for 2.5 MeV electrons in $\mathrm{PtCoO}_{2}$ [24], and thus 2-3 orders of magnitude larger than the typical thickness of our

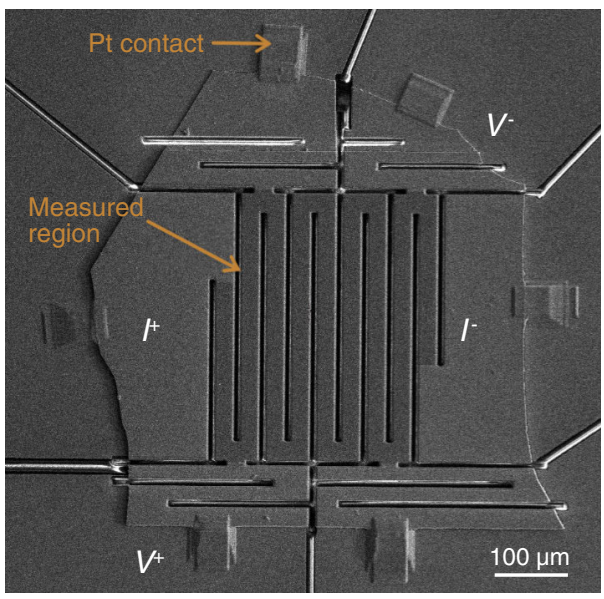

FIG. 5. SEM image of a microstructured $\mathrm{PtCoO}_{2}$ sample used for the in situ measurements of resistivity. The measured region of the device appears darker in the SEM image because the gold has been removed from it; the rest of the sample, the Pt contacts, and the substrate are covered with sputtered gold.

crystals. This further ensures that the majority of electrons can be measured using a Faraday cage placed behind the sample stage, enabling the monitoring of the current fluctuations during a measurement (Fig. 4).

The irradiation is paused at regular intervals to perform four-point in situ resistance measurements, and therefore monitor the increase of resistivity as a function of electron dose. Measuring resistivity of crystals as conductive and as small as delafossites in those demanding conditions presents additional challenges, which we addressed by developing dedicated sample preparation methods, as described below.

\section{Sample preparation}

In order to enable a reliable in situ measurement of the low-temperature resistivity of delafossites, we used focused ion beam sculpting to increase the effective length of the measured sample, as shown in Fig. 5. The standard method of sample mounting for FIB sculpting requires using a layer of glue to attach the crystal to a substrate, as was done in previous transport studies of delafossite metals $[18,20]$. However, glue degrades quickly in the electron beam, motivating us to use a glue-free mounting method instead. The sample, held on a $\sim 25-\mu$ m-thick mica substrate by the electrostatic force, was first covered with a 150-nmthick layer of sputtered gold. Pt contacts were then deposited in situ in the FIB, providing a mechanical connection between the sample and the gold-covered substrate. To achieve contact resistances on the order of $1 \Omega$, a second layer of gold was sputtered over the sample, substrate, and the Pt contacts. A crystal mounted in this way was FIB structured using the standard techniques described in detail in Ref. [25], including the removal of gold from the measured part of the device. This active 


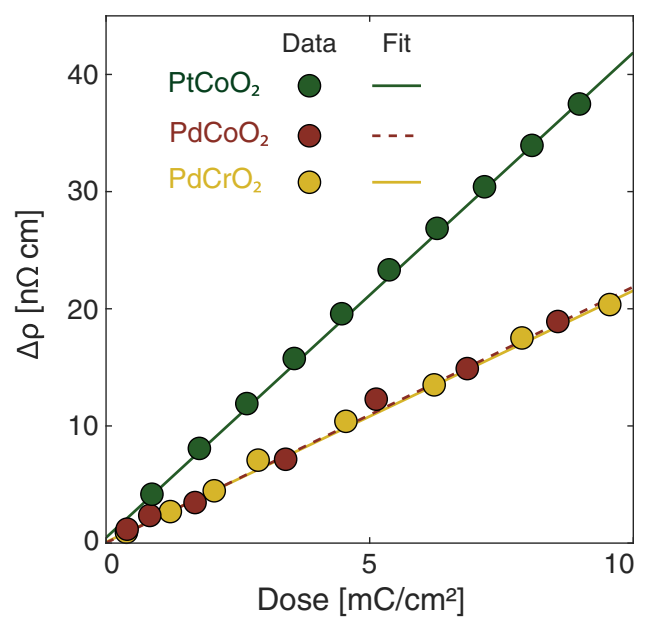

FIG. 6. Increase of resistivity as a function of electron dose for three delafossite metals: $\mathrm{PtCoO}_{2}, \mathrm{PdCoO}_{2}$, and $\mathrm{PdCrO}_{2}$.

region is shaped like a meander, whose width of $60 \mu \mathrm{m}$ was chosen to ensure that the edge scattering does not significantly contribute to the measured resistivity [18]. The length of the meander is approximately $3 \mathrm{~mm}$ in the example structure shown in Fig. 5. The two current contacts (labeled $I^{-}$and $I^{+}$in Fig. 5) and two of the voltage contacts $\left(V^{-}\right.$and $\left.V^{+}\right)$were used for the in situ measurement. Additional meanders were cut in all the voltage contacts so that the strain caused by the differential thermal contraction between the sample and the substrate can relax. Consequently, the samples were mechanically stable enough to allow for multiple iterations of irradiation and rapid thermal cycling. The $\mathrm{PtCoO}_{2}$ samples prepared in this way had low-temperature resistances in the range of 1 to $20 \mathrm{~m} \Omega$.

\section{B. Resistivity increase}

The increase of the resistivity of $\mathrm{PtCoO}_{2}, \mathrm{PdCoO}_{2}$, and $\mathrm{PdCrO}_{2}$ as a function of electron dose is shown in Fig. 6. In all three compounds the dependence of resistivity on dose is linear in the investigated range. The rate of resistivity increase is higher in $\mathrm{PtCoO}_{2}$ than in $\mathrm{PdCoO}_{2}$ and $\mathrm{PdCrO}_{2}$, which show the same rate of increase. These observations indicate that the resistivity is dominated by the defects in the conductive $\mathrm{Pt} / \mathrm{Pd}$ planes, as expected in such twodimensional systems: $\mathrm{Pd}$ defects are created at the same rate in $\mathrm{PdCoO}_{2}$ and $\mathrm{PdCrO}_{2}$, while the rate of defect introduction is higher in $\mathrm{PtCoO}_{2}$, because the larger nuclear charge of the $\mathrm{Pt}$ atoms leads to a stronger interaction with the incoming electrons. The higher rate of defect introduction is the reason we have chosen to perform further measurements on $\mathrm{PtCoO}_{2}$.

The fact that the resistivity increases as a function of electron dose indicates both that electron irradiation introduces defects and that those defects contribute to resistivity. Therefore, if the scattering of conduction electrons by defects is suppressed in delafossites, that suppression is not complete. However, the observations so far do not rule out a partial suppression of scattering, and therefore cannot be used to deduce how sensitive the resistivity of delafossites is to disorder. This requires quantifying the introduced defect concentration, which we have done using electronenergy-dependent measurements, as described in the following section.

\section{Quantifying the introduced Frenkel pair concentration}

In order to calculate the concentration of introduced Frenkel pairs, we adopt the approach used in the studies of electron irradiation of elemental metals and their alloys [26], as well as in the high-temperature superconductor YBCO [27] and graphene [28]. The method relies on the electron-energy dependence of the cross section for the formation of Frenkel pairs, $\sigma_{\mathrm{FP}}$. A quantity directly proportional to $\sigma_{\mathrm{FP}}$, such as the rate of increase of resistivity, is measured as a function of electron energy. This measurement is compared to a calculation of the electron-energy dependence of $\sigma_{\mathrm{FP}}$, revealing the factor relating the measured quantity and $\sigma_{\mathrm{FP}}$, therefore allowing the determination of the latter in absolute units.

This approach relies on the calculation of $\sigma_{\mathrm{FP}}$, which requires the knowledge of the cross section for the scattering of electrons off stationary nuclei and understanding of the conditions under which such a scattering event results in an atom being displaced from its equilibrium lattice site. If the incoming particles are nonrelativistic, the former is given by the well-known Rutherford expression for a differential scattering cross section:

$$
\frac{d \sigma_{R}}{d \Omega}=\left(\frac{Z e^{2}}{4 \pi \varepsilon_{0}} \frac{\hbar c}{4 E_{K}}\right)^{2} \frac{1}{\sin ^{4}\left(\frac{\vartheta}{2}\right)},
$$

where $E_{K}$ is the electron kinetic energy, $\vartheta$ the scattering angle, $Z$ the atomic number of the nucleus, $e$ the elementary charge, $\varepsilon_{0}$ the vacuum permeability, $\hbar$ the reduced Planck's constant, and $c$ the speed of light.

However, the high energy of the electrons in the present experiment requires the use of the relativistic extension of the Rutherford expression. Mott calculated the relativistic differential cross section for the scattering of electrons and pointlike nuclei using Darwin's solution to the Dirac equation [29,30]. His formula is exact, and it gives the cross section as a sum of two conditionally convergent infinite series. It therefore needs to be numerically evaluated for every individual case, motivating numerous approximate expressions for the Mott cross section, such as the commonly used McKinley-Feshbach cross section [31]. However, the McKinley-Feshbach approximation is only valid for atoms of the nuclear charge $Z<27$. Although it is often used for heavier atoms, such as copper [26], it is not expected to be suitable for Pd or Pt. Instead, 
we follow the approach given in Refs. [32,33], in which the ratio of the Mott and Rutherford cross section is expressed as a function of scattering angle $(\vartheta)$ and the ratio of the electron velocity to the speed of light $\beta[34]$ as

$$
\frac{\sigma_{M}}{\sigma_{R}}=\sum_{j=0}^{4} \sum_{k=1}^{6} b_{j k}(\beta-\bar{\beta})^{k-1}[1-\cos (\vartheta)]^{j / 2},
$$

where $\bar{\beta}=0.7181287 . b_{j k}$ are a set of 30 parameters, obtained by fits to the numerical solutions to Mott's equations. They are listed in Ref. [32] for all elements with $Z \leq 90$ and in Ref. [33] for all elements with $Z \leq 118$; for more details on the mathematics and numerics behind those calculations, see Refs. [32,33], and the references within. In this work, we calculate the Mott differential cross section using the expressions (1) and (2) combined with parameters $b_{j k}$ as listed in Ref. [33].

A scattering event described by the Mott cross section results in a Frenkel pair if, and only if, the energy transferred to the nucleus is larger than a threshold, the so-called displacement energy $\left(E_{d}\right)$. As long as the displaced atom does not introduce secondary defects, the total cross section for production of Frenkel pairs is given by

$$
\sigma_{\mathrm{FP}}=2 \pi \int_{\vartheta\left(E_{D}\right)}^{\pi} \frac{d \sigma_{M}}{d \Omega} \sin \vartheta d \vartheta
$$

where $\vartheta\left(E_{D}\right)$ denotes the scattering angle corresponding to the minimal energy transfer of $E_{d}$ [35].

The calculation described above has only one free parameter, the displacement energy. In Fig. 7 we show the cross section for production of $\mathrm{Pt}$ Frenkel pairs calculated for a range of displacement energies. For each of them there is a different well-defined minimum electron energy at which Frenkel pairs can be created. Consequently, the curves at different values of $E_{d}$ are not related by a simple scaling relation, and the displacement energy can be uniquely determined by a measurement of the electron-energy dependence of any quantity which is proportional to the Frenkel pair production cross section.

The rate of resistivity increase as a function of dose is such a quantity. We have therefore irradiated two samples of $\mathrm{PtCoO}_{2}$ using a range of electron energies between 0.8 and $2.5 \mathrm{MeV}$ [36]. As shown in Fig. 8(a), the resistivity increases more slowly when the electron energy is decreased, as expected. The slopes measured on the two samples are plotted as a function of electron energy in Fig. 8(b) (symbols). They were fitted to the Pt Frenkel pair production cross section, as calculated by the methods described above, determining the displacement energy to be equal to $E_{d}=10 \mathrm{eV}$. The good agreement of the data and the calculation both justifies the assumption that significant numbers of secondary defects are not created and confirms that the resistivity is dominated by Pt defects.

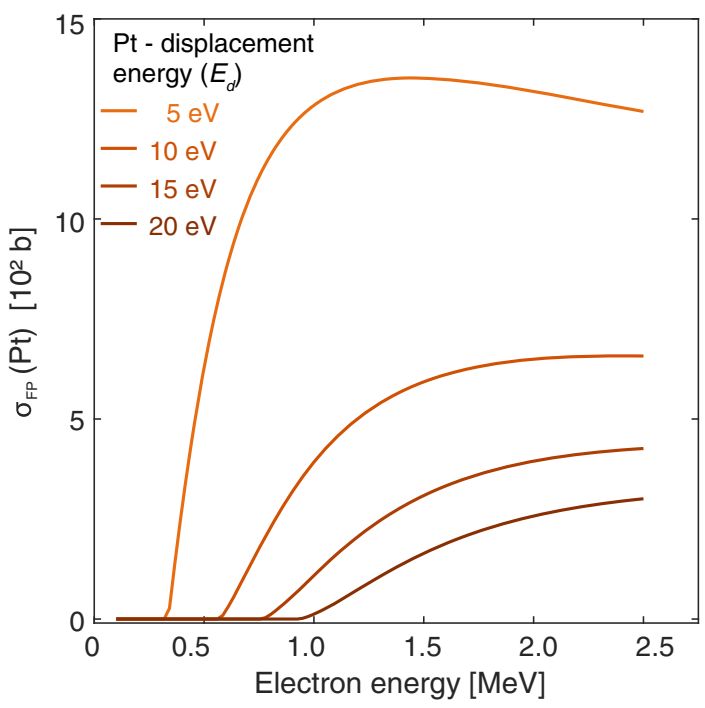

FIG. 7. Frenkel pair production cross section for Pt atoms as a function of electron energy, for a range of displacement energies.
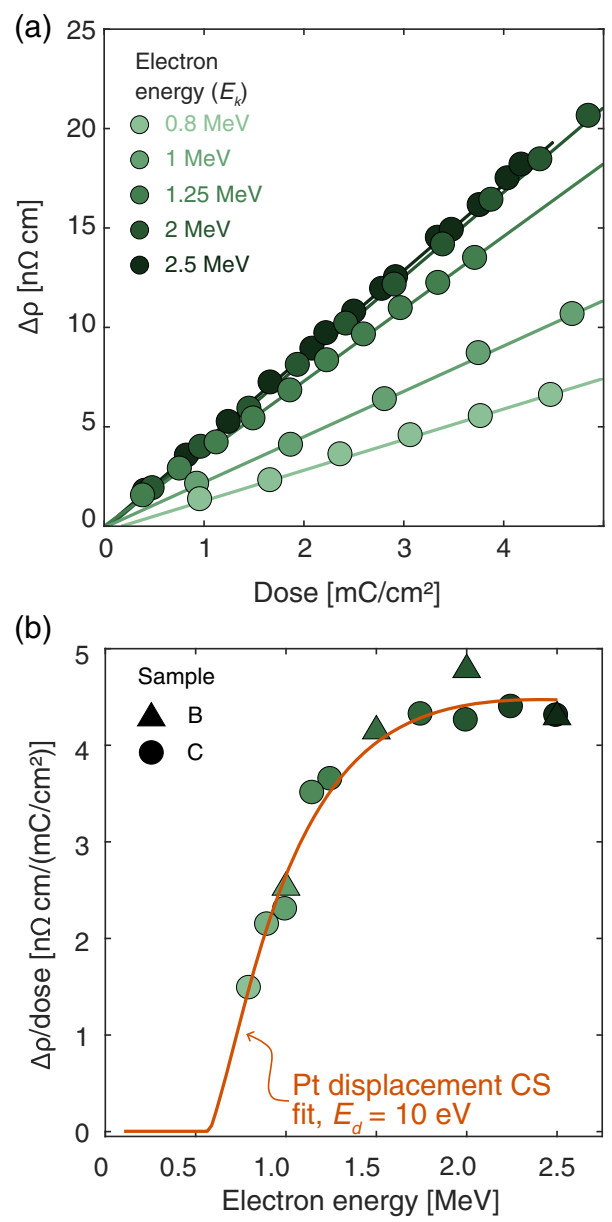

FIG. 8. (a) $\mathrm{PtCoO}_{2}$ resistivity as a function of electron dose for a range of electron energies. (b) The rate of increase of the resistivity of $\mathrm{PtCoO}_{2}$ as a function of electron energy, measured on two samples (symbols), compared to the calculated cross section (CS) for the displacement energy of $10 \mathrm{eV}$. 
What is more, it allows for a determination of the Frenkel pair production cross section in absolute units; for electrons of kinetic energy of $2.5 \mathrm{MeV}$, it is $\sigma_{\mathrm{FP}}(\mathrm{Pt}, 2.5 \mathrm{MeV})=$ $657 \mathrm{~b}$. Multiplying the irradiation dose by $\sigma_{\mathrm{FP}}$ and dividing it by the charge of an electron directly yields the introduced Frenkel pair concentration.

\section{RESISTIVITY AS A FUNCTION OF FRENKEL PAIR CONCENTRATION}

In Fig. 9(a) we show the dependence of resistivity on the concentration of Frenkel pairs introduced by electron irradiation for four $\mathrm{PtCoO}_{2}$ samples microstructured into long meanders, as shown in Fig. 5. The dependence is linear in all the samples, with a slope of $(9.1 \pm 0.2) \times 10^{3} \mathrm{n} \Omega \mathrm{cm} / \%$. The residual resistivity of the samples prior to irradiation, however, varied between 40 and $65 \mathrm{n} \Omega \mathrm{cm}$, reflecting different defect concentration in as-grown crystals. Assuming that
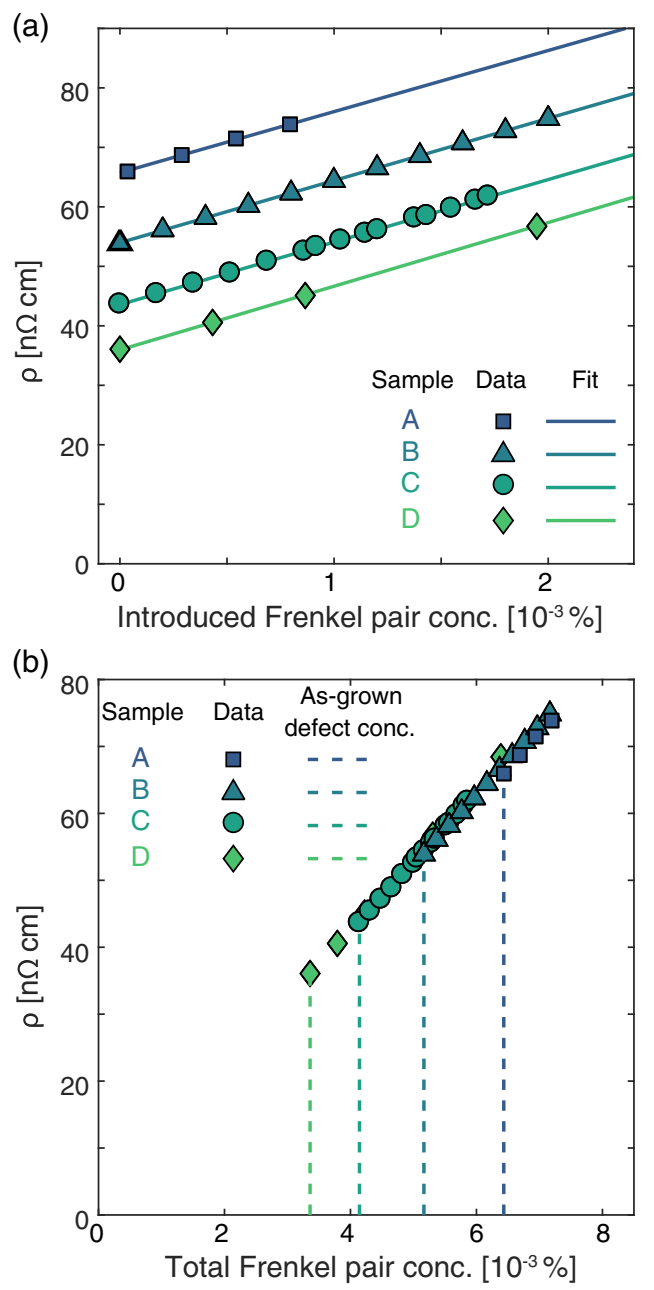

FIG. 9. Resistivity of four $\mathrm{PtCoO}_{2}$ samples as a function of (a) introduced defect concentration and (b) the total defect concentration, estimated from the interpolation of lines in (a). The dashed lines indicate the effective Frenkel pair concentration in the samples before irradiation. all the defects that contribute to resistivity are Frenkel pairs, our measurements allow us to estimate their concentration prior to irradiation by extrapolating the lines in Fig. 9(a) to zero resistivity. Once plotted as a function of total Frenkel pair concentration, found by adding the initial and the introduced defect concentrations, the resistivities of all the samples collapse on the same curve, as shown in Fig. 9(b). The estimated initial Frenkel pair concentrations, indicated by the dashed lines, range between $0.004 \%$ and $0.007 \%$ in the investigated samples, while the lowest low-temperature resistivity reported in $\mathrm{PtCoO}_{2}(20 \mathrm{n} \Omega \mathrm{cm})$ [20] corresponds to a defect concentration of $0.002 \%$. The assumption that all the defects contributing to resistivity are Frenkel pairs is certainly not entirely accurate, but other point defects in the Pt layers are not expected to have a significantly different influence on resistivity. The estimate for the initial point defect concentration obtained in this way should therefore be correct to within a factor of 2-3.

\section{A. Comparison with other 2D materials}

In addition to enabling the determination of the purity of as-grown crystals, the analysis presented in the previous section allows the sensitivity of the delafossite resistivity to point defects to be compared to that of other materials. The sheet resistivity $\rho^{2 \mathrm{D}}$ of $2 \mathrm{D}$ materials is usually assumed to depend on the ratio of the in-plane defect concentration $n_{d}$ and carrier concentration $n$ as

$$
\rho_{\mathrm{unit}}^{2 \mathrm{D}}=\frac{4 \hbar}{e^{2}} \frac{n_{d}}{n},
$$

where $e$ is the electron charge. This expression is referred to as the "unitary limit" and physically corresponds to the case of the strongest possible $s$-wave scattering; for an outline of the derivation, see the Appendix A. It is empirically known that the in-plane resistivity of several layered materials follows the unitary scattering prediction to a precision of $\sim 30 \%$. This is true of $\mathrm{Sr}_{2} \mathrm{RuO}_{4}$ [37], $\mathrm{La}_{2-x} \mathrm{Sr}_{x} \mathrm{CuO}_{4}$, and $\mathrm{YBa}_{2} \mathrm{Cu}_{3} \mathrm{O}_{7-y}$ [38], in which the defects are introduced by substitution ( $\mathrm{Ti}$ for $\mathrm{Ru}$ in $\mathrm{Sr}_{2} \mathrm{RuO}_{4}, \mathrm{Zn}$ for $\mathrm{Cu}$ in the cuprates), as well as of electron-irradiated $\mathrm{YBa}_{2} \mathrm{Cu}_{3} \mathrm{O}_{7-y}$ [39]. A comparison of our measurements with the unitary scattering limit is therefore simultaneously a comparison with other layered materials; a strong scattering suppression in $\mathrm{PtCoO}_{2}$ would correspond to a strong deviation from the unitary scattering prediction.

To test if this is the case, in Fig. 10 we compare the resistivity increment in several samples of the three delafossites, $\mathrm{PtCoO}_{2}, \mathrm{PdCoO}_{2}$, and $\mathrm{PdCrO}_{2}$, to the unitary scattering prediction. The introduced $\mathrm{Pd}$ Frenkel pair concentration in $\mathrm{PdCoO}_{2}$ and $\mathrm{PdCrO}_{2}$ is calculated assuming that the displacement energy for $\mathrm{Pd}$ is the same as that measured for $\mathrm{Pt}, 10 \mathrm{eV}$, yielding a Pd Frenkel pair production cross section of $\sigma_{\mathrm{FP}}(\mathrm{Pd}, 2.5 \mathrm{MeV})=315 \mathrm{~b}$. The point defect concentration $n_{d}$ in Eq. (4) is taken to 


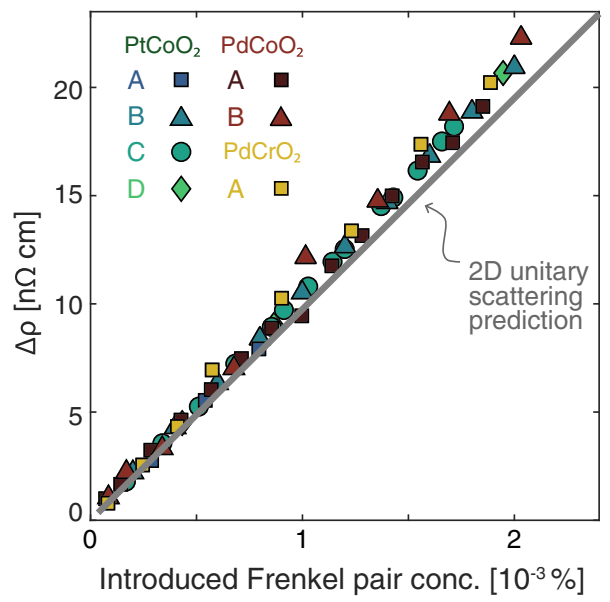

FIG. 10. Resistivity increment of four $\mathrm{PtCoO}_{2}$ samples, two $\mathrm{PdCoO}_{2}$ samples and a $\mathrm{PdCrO}_{2}$ sample as a function of introduced Frenkel pair concentration compared to the unitary scattering prediction.

be equal to the introduced Frenkel pair concentration. The striking agreement of the resistivity increment and the unitary prediction demonstrates that the resistivity of delafossites is as sensitive to defects as that of other layered materials. This analysis conclusively shows that the enormous low-temperature mean free paths of the delafossite metals are caused by their unusual purity.

It is worth emphasizing that the unitary scattering prediction does not depend on any free parameters, and the only fitting parameter leading to the conclusion drawn from Fig. 10 is the displacement energy, which has been independently experimentally determined, as described in Sec. III C. The analysis, however, equates the concentration of strong in-plane scatterers with that of Frenkel pairs and assumes that the resistivity arises only from those in-plane scatterers. This cannot be exactly true, because each Frenkel pair consists of both an in-plane vacancy and an interstitial, which is likely to be out of plane. The former are strong in-plane scatterers for which the unitary scattering is expected to be applicable, while the latter scatter more weakly. In practice, the resistivity is increased due to both of them, suggesting that the added resistivity per in-plane vacancy is smaller than suggested by the unitary scattering. However, even in the extreme and nonphysical case of outof-plane interstitials adding as much resistivity as the inplane vacancies, the resistivity would be suppressed by at most a factor of 2 from the unitary value. This in no way changes the conclusion that the extremely long mean free paths in delafossites arise mainly from their remarkable purity.

The excellent agreement between the measurements on the three compounds indicates that the large difference in the resistivity increment as a function of electron dose shown in Fig. 6 can be attributed solely to the lower differential cross section for the formation of Pd defects, and that the resistivity of the three compounds is equally sensitive to defects. This allows for an estimate of the Frenkel pair concentration in the as-grown crystals of $\mathrm{PdCoO}_{2}$ : the residual resistivity in the purest crystals reported to date is $8.1 \mathrm{n} \Omega \mathrm{cm}$ [20], corresponding to an estimated point defect concentration of $0.001 \%$.

\section{FIRST-PRINCIPLES CALCULATIONS}

As discussed above, the irradiation studies indicate that the low resistivity of $\mathrm{PdCoO}_{2}$ and $\mathrm{PtCoO}_{2}$ results from an unusually low defect density in the as-grown materials. In order to explore this theoretically, we use density functional theory (DFT) to calculate properties of Pd/Pt-related native defects in $\mathrm{PdCoO}_{2}$ and $\mathrm{PtCoO}_{2}$ including vacancies, interstitials, and Frenkel pairs. For computational details, see Appendix B.

\section{A. Formation energy comparison}

As a result of configurational entropy, there is always a finite concentration of native point defects in a crystal in thermodynamic equilibrium. Additional imperfections may exist as a result of kinetic barriers that prevent the system from reaching thermal equilibrium. We would like to determine whether the reduced defect concentrations observed in $\mathrm{PdCoO}_{2}$ and $\mathrm{PtCoO}_{2}$ are a result of the intrinsically higher formation energy of $\mathrm{Pd} / \mathrm{Pt}$-related defects or are from growth-specific kinetic factors.

The defect concentration is given by the formation free energy of the defect $[40,41]$. However, this quantity is computationally very intensive to determine accurately in complex materials. Thus our strategy will be to compare the formation energy (i.e., we neglect electronic and vibrational entropy) of Pd-related defects in $\mathrm{PdCoO}_{2}$ with those in $\mathrm{Pd}$ metal, in order to determine if they are significantly larger and could explain the low defect concentrations in the delafossite metals. The formation energy of defect $X$ in a metal is given by

$$
E_{\text {form }}[X]=E_{\text {tot }}[X]-\left(E_{\text {tot }}[\mathrm{bulk}]+\sum_{i} n_{i} \mu_{i}\right),
$$

where $E_{\text {tot }}[X]$ is the energy of a supercell containing the defect $X, E_{\text {tot }}[$ bulk $]$ is the energy of a bulk cell of corresponding size, $n_{i}$ is the number of atomic species $i$ added or removed to create the defect, and $\mu_{i}$ is the chemical potential of species $i . E_{\text {tot }}[X]$ and $E_{\text {tot }}[\mathrm{bulk}]$ are calculated using DFT, while the chemical potential(s) $\mu_{i}$ are specific to the experimental growth or annealing conditions. By enforcing stability conditions, we can obtain theoretical limits on $\mu$, which can provide guidance as to the possible experimental conditions; for the calculations below, we assume Pd-rich (i.e., O-poor) conditions (see Appendix C). 
TABLE I. Formation energies in $\mathrm{eV}$ for Pd-related native defects in $\mathrm{PdCoO}_{2}$ and $\mathrm{Pd}$ metal. In parentheses are previous DFT GGA calculations.

\begin{tabular}{lcl}
\hline \hline Defect & $\mathrm{PdCoO}_{2}$ & \multicolumn{1}{c}{$\mathrm{Pd}$} \\
\hline Pd vacancy & 0.88 & $1.26\left(1.20^{\mathrm{a}}, 1.19^{\mathrm{b}}\right)$ \\
Pd interstitial & 6.28 & $3.82\left(3.43^{\mathrm{c}}\right)$ \\
Pd Frenkel pair & 7.11 & 5.11 \\
\hline \hline
\end{tabular}

${ }^{\mathrm{a}}$ Ref. [43].

${ }^{b}$ Ref. [44].

${ }^{c}$ Ref. [42].

In Table I we compare the energies of Pd vacancies, interstitials, and Frenkel pairs in $\mathrm{PdCoO}_{2}$ with those in elemental Pd. Our formation energy for the Pd vacancy and self-interstitial in Pd metal are consistent with previous calculations [42-44] and a slight underestimation compared to the experimental range of 1.5-1.85 eV [44-46]. The general behavior is similar between $\mathrm{Pd}$ metal and $\mathrm{PdCoO}_{2}$, with vacancies expected to be the dominant defect. The formation energy of interstitials in $\mathrm{PdCoO}_{2}$ is significantly larger that in Pd; however, in both cases, it is not expected that interstitials will be present in large concentrations. The large formation energy of interstitials is expected in close-packed metals. For $\mathrm{PdCoO}_{2}$ it is likely due to the short $\mathrm{Pd}-\mathrm{O}$ bond lengths of $\sim 2 \AA$ (cf. $2.8 \AA$ for the $\mathrm{Pd}-\mathrm{Pd}$ bonds in $\mathrm{PdCoO}_{2}$ ), forcing the interstitial atom unfavorably close to the Pd layer (for a more detailed discussion of the interstitial structure, see Appendix D).

The Frenkel pair formation energy in both cases is approximately the sum of the vacancy and interstitial energies. Thus, there is not a significant energy gain from having the interstitial in the vicinity of the vacancy, and we find that in the delafossites, the interstitial needs to be placed several lattice sites away to prevent it from relaxing back to the vacancy site (see Sec. V B).

We find similar behavior between $\mathrm{PtCoO}_{2}$ (not shown) and $\mathrm{PdCoO}_{2}$. We therefore find no evidence that the exceptionally low defect concentrations seen in as-grown delafossites are a result of uncharacteristically high formation energies for $\mathrm{Pd} / \mathrm{Pt}$ defects in those materials.

\section{B. Displacement energy}

Of course, the formation energy of Frenkel pairs calculated in Sec. VA does not apply to the nonequlibrium creation of defects via irradiation; the important quantity in this case is the displacement energy. To obtain a theoretical estimate of the displacement energy, we calculate the adiabatic energy barrier for a $\mathrm{Pt} / \mathrm{Pd}$ atom to be displaced from its position in the lattice toward an interstitial site using the nudged elastic band method.

The results are shown in Fig. 11, where the initial structure is the ideal bulk structure, and the final structure is that of the relaxed Frenkel pair. We can see that for both

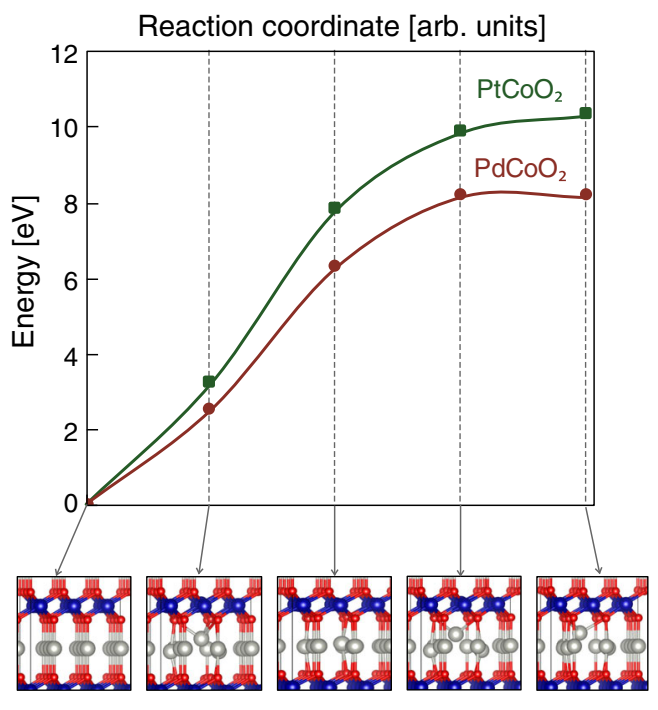

FIG. 11. Adiabatic barrier for Frenkel pair formation in $\mathrm{PdCoO}_{2}$ and $\mathrm{PtCoO}_{2}$, from nudged elastic band DFT calculations.

$\mathrm{PdCoO}_{2}$ and $\mathrm{PtCoO}_{2}$, there is essentially no additional kinetic barrier to the formation: the energy required to adiabatically create the Frenkel pair is approximately equal to its formation enthalpy, i.e., $\sim 8 \mathrm{eV}$ (see Appendix B) in $\mathrm{PdCoO}_{2}$ and $\sim 10 \mathrm{eV}$ in $\mathrm{PtCoO}_{2}$. It is important to note that the total energy of the barrier should be considered as a lower bound for the displacement energy, as it allows for all degrees of freedom perpendicular to the reaction coordinate to relax, which will likely not occur in the actual Mott scattering process. The results are therefore fully consistent with the value deduced from experiment of $10 \mathrm{eV}$.

\section{INFLUENCE OF DEFECTS ON TRANSPORT PROPERTIES}

All of the experiments and analysis shown so far address the question of the influence of defects on the lowtemperature resistivity of delafossite metals. It is often implicitly assumed that the various contributions to resistivity are additive, obeying the so-called Matthiessen rule. Measuring the transport properties of a sample before and after electron irradiation offers a unique opportunity to test this assumption in a controlled manner. Such a study is, however, by no means guaranteed to succeed. First of all, as the sample is warmed up from the irradiation temperature of $22 \mathrm{~K}$, the defects become more mobile and may recombine, therefore removing any added resistivity. Even if the defects remain in the sample by room temperature, they may slowly anneal, leading to time-dependent resistivity. In $\mathrm{PtCoO}_{2}$, the added resistivity does decrease during the initial warm-up to room temperature to $\sim 65 \%$ of its original value. However, the remaining resistivity stays unchanged at room temperature and during subsequent 


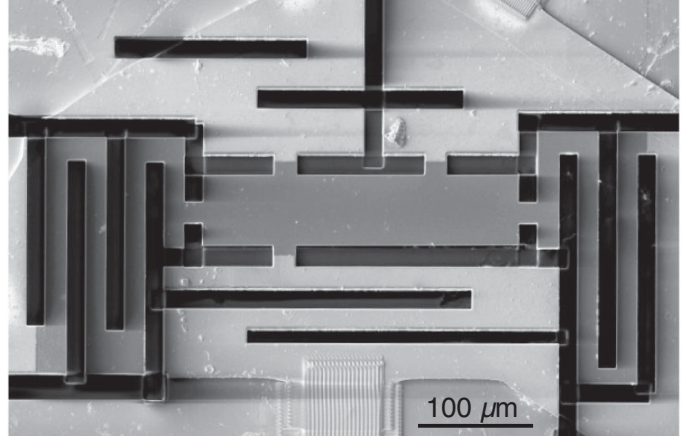

FIG. 12. SEM image of a microstructured $\mathrm{PtCoO}_{2}$ sample used for the ex situ measurements of the temperature- and magnetic field-dependent resistivity.

thermal cycles to low temperatures (for more details, see Appendix E), enabling a controlled and reproducible study of the disorder influence on transport. Such a study is beyond the scope of this work, but we show below two intriguing results which point to its broad significance.

In Fig. 12 we show an SEM image of a sample we have structured for the temperature- and magnetic field-dependent measurements. Its well-defined bar geometry enables a precise determination of its resistivity, which prior to irradiation agrees quantitatively with the published data [20]. The estimated defect concentration of the sample before irradiation was $0.0046 \%$, while after the irradiation to a total dose of $188 \mathrm{mC} / \mathrm{cm}^{2}$ by electrons of $2.5 \mathrm{MeV}$ kinetic energy, and a subsequent warm-up to room temperature, it was increased by nearly an order of magnitude, to $0.038 \%$.

In Fig. 13 we show the resistance of the sample shown in Fig. 12 as a function of temperature [Fig. 13(a)] and magnetic field [Fig. 13(b)], before and after irradiation. The temperature dependence of resistivity remains nearly unchanged, with the defects adding an approximately temperature-independent offset, indicating a good agreement with Matthiessen's rule.

Introduced defects reduce the overall scale of magnetoresistance by a factor of 2.3. Again, this reduction can be qualitatively understood within the semiclassical picture of magnetoresistance, assuming independent scattering events. Finite magnetoresistance indicates the presence of more than one length scale governing transport, and is proportional to the square of their difference [47]. In a single band material of nearly constant Fermi velocity [20], this suggests at least two different scattering rates at different points of the Fermi surface, $\tau_{1}$ and $\tau_{2}=\alpha \tau_{1}$; the magnetoresistance is proportional to $\left(\tau_{1}-\tau_{2}\right)^{2}=$ $(1-\alpha)^{2} \tau_{1}^{2}$. If Matthiessen's rule is obeyed and the defect scattering exhibits the same $\mathbf{k}$ dependence as $\tau_{1}$ and $\tau_{2}$ (certainly a good approximation at low temperatures), the scattering times are changed by defects into $\tau_{1 d}$ and
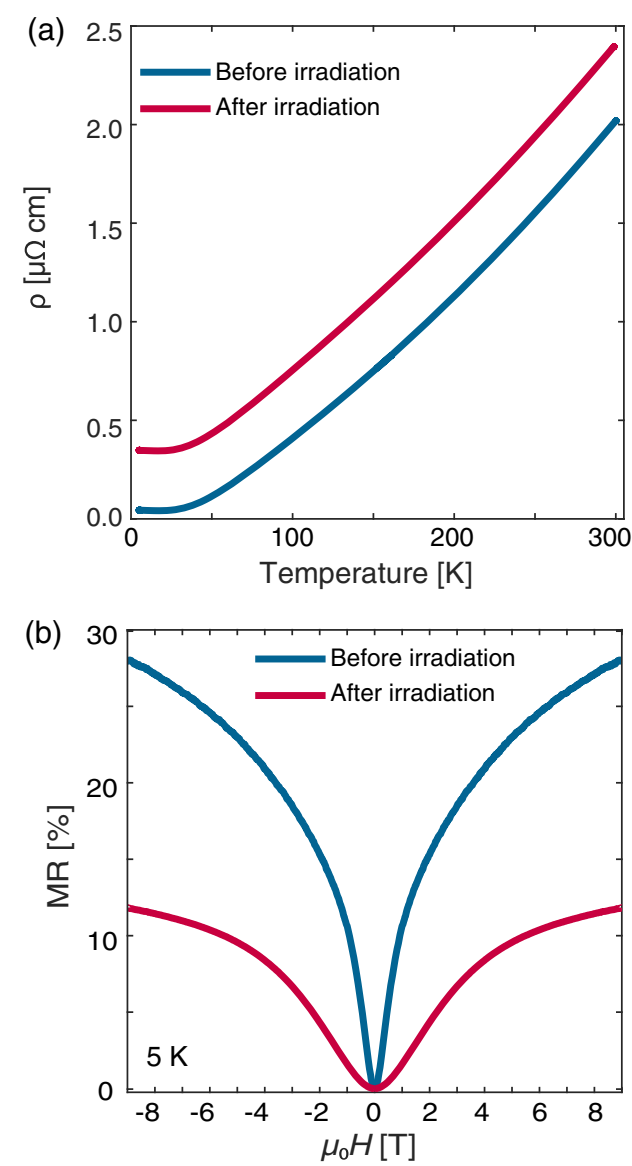

FIG. 13. The (a) temperature-dependent resistivity and (b) magnetoresistance $[\mathrm{MR}=[\rho(H)-\rho(0)] / \rho(0)]$ of the sample shown in Fig. 12 before and after irradiation.

$\tau_{2 d}=\alpha \tau_{1 d}$. The magnetoresistance is then proportional to $\left(\tau_{1 d}-\tau_{2 d}\right)^{2}=(1-\alpha)^{2} \tau_{1 d}^{2}$. The defects reduce the scattering times $\left(\tau_{1 d}<\tau_{1}\right)$, so this represents a reduction in magnetoresistance compared to the case without the added defects. Therefore, regardless of the cause of the different scattering rates across the Fermi surface, adding a source of scattering to all the states decreases magnetoresistance, as observed.

Although at first sight these observations are not unusual, a more detailed analysis offers additional insight, as discussed in the following section.

\section{DISCUSSION}

The experimental data and analysis presented in this paper provide very strong evidence in favor of a remarkable experimental fact: the extremely long low-temperature mean free paths of metallic delafossites are mainly due to an exceptional level of crystalline perfection of the Pd or Pt planes in which the conduction takes place. We are able to deduce point defect levels as low as $0.001 \%$ in the best as-grown crystals. 
(a)
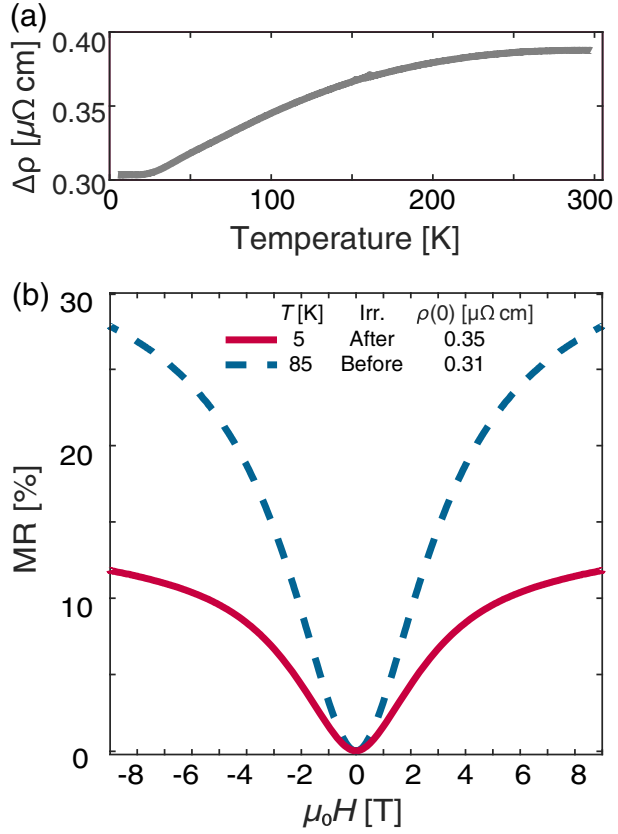

FIG. 14. (a) The difference of the temperature-dependent resistivity after and before the irradiation. (b) The magnetoresistance $[\mathrm{MR}=[\rho(H)-\rho(0)] / \rho(0)]$ measured at $5 \mathrm{~K}$ after irradiation compared to that measured at $85 \mathrm{~K}$ before irradiation (dashed line). The zero field resistivity (legend) was the same within $10 \%$ in the two measurements.

The level of control of defect densities that we have demonstrated, combined with the data quality obtainable from the microstructures that we have used, will also open paths to new discoveries. For example, we will be able to carry out a stringent examination of subtle effects that challenge understanding of scattering of electrons in solids. Although the agreement with Matthiessen's rule, shown in Fig. 13(a), looks excellent on first inspection, more detailed analysis demonstrates a systematic, temperature-dependent deviation, as seen in the plot of the difference between the temperature-dependent resistivity of the irradiated and pristine sample [Fig. 14(a)]. This suggests that the scattering from the in-plane defects that we have introduced is not entirely independent of electron-phonon scattering. The data shown in Fig. 14(b) offer further information on the interplay of the two sources of scattering. At $5 \mathrm{~K}$ after irradiation, the resistivity is almost identical to that at $85 \mathrm{~K}$ in the as-grown crystal, so the resistive mean free paths averaged around the Fermi surface are also very similar. However, the magnetoresistance, which is sensitive to the $\mathbf{k}$ dependence of the mean free path around the Fermi surface [48], differs in scale by over a factor of 2. The Fermi velocity is $\mathbf{k}$ independent to within a few percent [20], so mean free path variations must be due to variations in the scattering rates, indicating that the $\mathbf{k}$ dependence of the scattering rate depends on the scattering mechanism. These observations strongly motivate future work to study the magnetotransport as a function of temperature and defect density, and the extent to which such data can be modeled using modern quantum transport theories [49] and calculations of the $\mathbf{k}$ dependence of the electron-phonon interaction [50].

Another avenue for future work that the current findings will stimulate is closer investigation of other materials that crystallize in the delafossite structure. Is the crystalline perfection that we have uncovered unique to the $\mathrm{Pt}$ and $\mathrm{Pd}$ layers of $\mathrm{PdCoO}_{2}, \mathrm{PdCrO}_{2}$, and $\mathrm{PtCoO}_{2}$, or might it occur in other delafossites as well? In particular, it will be interesting to investigate the crystalline purity of nonmetals from this structural class; this might be a route to the development of a new family of high-mobility semiconductors. We note, however, that the in-plane purity is sufficient to achieve a high conductivity in delafossite metals, because the out-of-plane impurities are very efficiently screened; this is proven by the data shown in Fig. 6 and their analysis in Fig. 10. In contrast, a high-mobility semiconducting delafossite would require crystalline perfection in all its layers, as the screening of out-of-plane defects would be less efficient [51]. While at present the level of disorder in the Co layers of $\mathrm{PdCoO}_{2}$ and $\mathrm{PtCoO}_{2}$ is not known, we confirm it to be below the resolution of electron microscopy (Fig. 3). Combined with the remarkable purity of the Pd and Pt layers we have uncovered, this motivates careful research on close structural relatives of these fascinating compounds.

\section{CONCLUSIONS}

In this paper we have used electron beam irradiation to introduce Frenkel pairs into the crystal structure of the delafossite metals $\mathrm{PdCoO}_{2}, \mathrm{PdCrO}_{2}$, and $\mathrm{PtCoO}_{2}$, to investigate the reason for their extremely long low-temperature mean free paths. By studying three compounds in which the cations change between $\mathrm{Pd}$ and $\mathrm{Pt}$, and $\mathrm{Co}$ and $\mathrm{Cr}$, we have demonstrated empirically that the resistivity is sensitive to defects in the $\mathrm{Pd} / \mathrm{Pt}$ layers. Varying the energy of the incident electrons between 0.8 and $2.5 \mathrm{MeV}$ produces data that can be fitted very well with Mott scattering theory with only one free fit parameter, which is independently confirmed by first-principles electronic structure calculations. The excellent fit to the Mott theory enabled quantitative measurement of the Frenkel pair production cross sections and hence the determination of the defect density of each irradiated crystal. The additional scattering thus introduced is in quantitative agreement with so-called unitary scattering, and the data allowed a reliable estimate of the density of point defects in as-grown crystals. This is as low as $0.001 \%$, proving that the main reason for the long mean free paths is a level of crystalline perfection rarely seen in multicomponent oxides. These findings raise 
important questions about the levels of purity potentially attainable in nonmetallic delafossites, and motivate further investigation of this intriguing class of materials.

The research data supporting this publication can be accessed at [52].

\section{ACKNOWLEDGMENTS}

We thank Olivier Cavani for operating the SIRIUS accelerator, C. Hooley, P. D. C. King, and R. Moessner for useful discussions, and A. Georges for suggesting complementing the experiments with the first-principles calculations. We acknowledge support from the Max Planck Society. V.S. and P. H. M. acknowledge EPSRC for $\mathrm{Ph} . \mathrm{D}$. studentship support through Grant No. EP/ L015110/1. E. Z. acknowledges support from the International Max Planck Research School for Chemistry and Physics of Quantum Materials (IMPRS-CPQM). Irradiation experiments performed on the SIRIUS platform were supported by the French National Network of Accelerators for Irradiation and Analysis of Molecules and Materials (EMIR\&A) under Project No. EMIR 2019 18-7099. Electron Microscopy at Cornell is supported by the U.S. National Science Foundation [Platform for the Accelerated Realization, Analysis, and Discovery of Interface Materials (PARADIM)] under Cooperative Agreement No. DMR-1539918, and DMR-1719875. The Flatiron Institute is a division of the Simons Foundation.

V.S. and P. H. M. contributed equally to this work.

\section{APPENDIX A: UNITARY SCATTERING LIMIT IN 3D AND 2D}

Within the Drude model the resistivity of a 3D material is given by

$$
\rho^{3 \mathrm{D}}=\frac{m_{\mathrm{eff}}}{n e^{2}} \frac{1}{\tau_{\mathrm{tr}}}=\frac{m_{\mathrm{eff}}}{n e^{2}} n_{d} v_{F}\left\langle\sigma_{\mathrm{imp}}\right\rangle,
$$

where $m_{\text {eff }}$ is the effective mass of the carriers, $n$ their concentration, $v_{F}$ the Fermi velocity, $n_{d}$ the defect concentration, and $\tau_{\text {tr }}$ the transport lifetime. $\left\langle\sigma_{\text {imp }}\right\rangle$ is the cross section for scattering of electrons off impurities, weighted by the $(1-\cos \vartheta)$ term accounting for the larger contribution to resistivity of backscattering compared to smallangle scattering.

If $\left\langle\sigma_{\text {imp }}\right\rangle$ is calculated using partial wave analysis assuming a central potential, it is found to be equal to [53]

$$
\left\langle\sigma_{\text {imp }}\right\rangle=\frac{4 \pi}{k^{2}} \sum_{l=0}^{\infty}(l+1) \sin ^{2}\left(\delta_{l}-\delta_{l+1}\right),
$$

where $k$ is the wave vector of the electrons and $\delta_{l}$ is the phase shift associated with the $l$ th Legendre polynomial comprising the electron wave function. The unitary limit corresponds to the strongest possible $s$-wave scattering, in which $\delta_{0}=\pi / 2$ and all other $\delta_{l}=0$. The resistivity in the unitary limit is then given by

$$
\rho_{\text {unit }}^{3 \mathrm{D}}=\frac{4 \pi \hbar n_{d}}{n e^{2} k_{F}},
$$

where $k_{F}$ is the Fermi wave vector.

Resistivity of a two-dimensional system is given by an expression analogous to Eq. (A1), but the 3D scattering area $\left\langle\sigma_{\text {imp }}\right\rangle$ has to be replaced by a $2 \mathrm{D}$ scattering length $\left\langle\lambda_{\text {imp }}\right\rangle$, given by [54]

$$
\left\langle\lambda_{\text {imp }}\right\rangle=\frac{4}{k}\left(1+2 \sum_{m=1}^{\infty} \sin ^{2} \delta_{m}\right) .
$$

In the unitary limit the scattering length is reduced to $\left\langle\lambda_{\text {imp }}\right\rangle=4 / k$; it is therefore comparable to the Fermi wavelength. The resistivity is given by

$$
\rho_{\text {unit }}^{2 \mathrm{D}}=\frac{4 \hbar}{e^{2}} \frac{n_{d}}{n},
$$

where we used $m_{\mathrm{eff}} v_{F}=\hbar k_{F}$.

\section{APPENDIX B: COMPUTATIONAL DETAILS}

Density functional theory calculations were performed using the generalized gradient approximation (GGA) of Perdew, Burke, Ernzerhof (PBE) [55], implemented in the VASP code [56]. It was shown in Ref. [57] that the experimental electronic structure [14] can be accurately reproduced with GGA $+U$ with the Hubbard $U=4 \mathrm{eV}$ on the $\mathrm{Pd} / \mathrm{Pt} d$ states, as well as spin-orbit coupling (SOC) included; however, the addition of a Hubbard $U$ and SOC

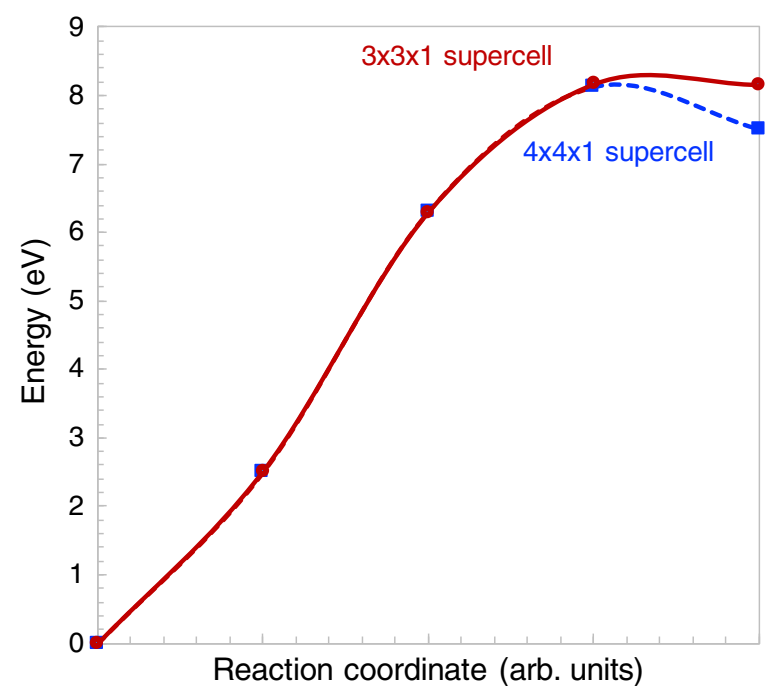

FIG. 15. Frenkel barrier dependence on supercell size for $\mathrm{PdCoO}_{2}$. 
have relatively minor effects on the electronic structure, and were thus neglected in this study. The experimental lattice constants (for the conventional cell) of $a=2.830 \AA, c=$ $17.743 \AA\left(\mathrm{PdCoO}_{2}\right)$ [10], and $a=2.823 \AA, c=17.808 \AA$ $\left(\mathrm{PtCoO}_{2}\right)$ [14] were used throughout.

A $520 \mathrm{eV}$ cutoff for the plane-wave basis set was used. A supercell of $5 \times 5 \times 1$ conventional cells (300 atoms) was used to calculate the formation energies of defects in the delafossites, with a $2 \times 2 \times 2$ gamma-centered $k$ mesh. For Pd metal, a $4 \times 4 \times 4$ supercell and $4 \times 4 \times 4 k$ mesh was used. Diffusion barriers and Frenkel pair formation barriers were calculated using the nudged elastic band method; due to the computational intensive nature of these calculations, a $3 \times 3 \times 1$ cell (and $3 \times 3 \times 1 k$ mesh) was used. The relatively small in-plane area of the supercell results in an overestimation of the energy of the separated Frenkel pair, as we demonstrate in Fig. 15 for $\mathrm{PdCoO}_{2}$, but does not affect our estimate of the barrier for Frenkel pair formation.

\section{APPENDIX C: LIMITS ON THE CHEMICAL POTENTIALS}

Under thermodynamic equilibrium the chemical potentials in Eq. (5) are limited by the stability of the bulk

(a)

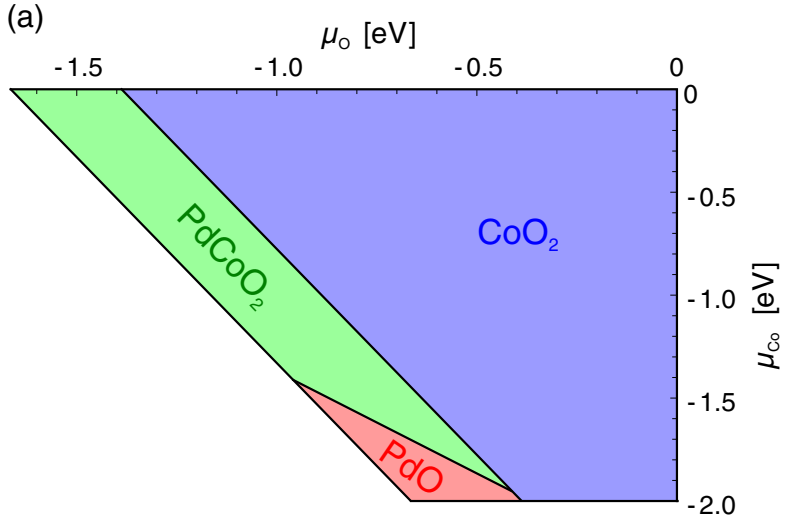

(b)

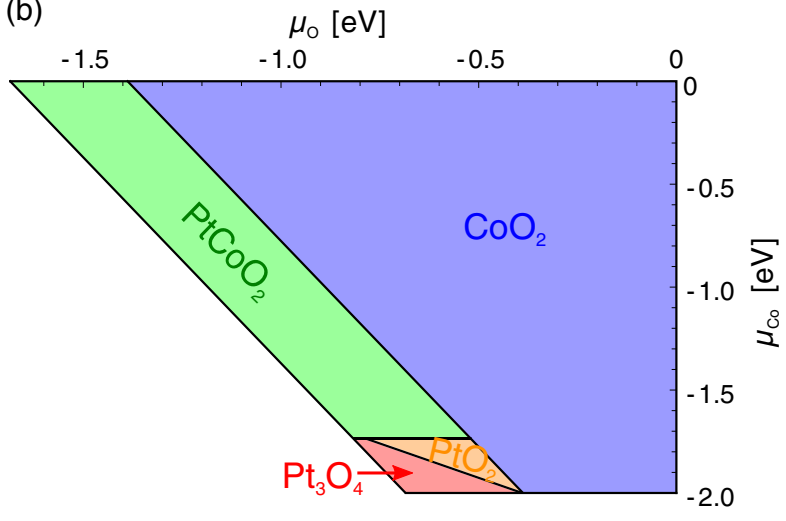

FIG. 16. $T=0$ stability diagram for (a) $\mathrm{PdCoO}_{2}$ and (b) $\mathrm{PtCoO}_{2}$ with respect to $\mathrm{O}$ and $\mathrm{Co}$ chemical potentials. Green region corresponds to chemical potentials where the delafossites are stable. material. We illustrate this for the case of $\mathrm{PdCoO}_{2}$. If we reference the chemical potentials to the corresponding elemental phases (i.e., the elemental metals for Pd and $\mathrm{Co}$, and an isolated $\mathrm{O}_{2}$ molecule for $\mathrm{O}$ ), the stability condition of $\mathrm{PdCoO}_{2}$ can be written [41] as

$$
\mu_{\mathrm{Pd}}+\mu_{\mathrm{Co}}+2 \mu_{\mathrm{O}}=\Delta H_{f}\left(\mathrm{PdCoO}_{2}\right),
$$

where $\Delta H_{f}\left(\mathrm{PdCoO}_{2}\right)$ is the enthalpy of formation of $\mathrm{PdCoO}_{2}$. Thus, only two chemical potentials can be varied independently; fixing those values will specify the third.

The range of chemical potentials during growth will be further limited by the formation of other phases with different stoichiomentry. In particular, we find that if conditions are too Co poor and/or O poor, $\mathrm{PdO}$ will form over $\mathrm{PdCoO}_{2}$ (equivalently, $\mathrm{Pt}_{3} \mathrm{O}_{4}$ over $\mathrm{PtCoO}_{2}$ ), and if the conditions are too $\mathrm{O}$ rich and/or $\mathrm{Co}$ rich, $\mathrm{CoO}_{2}$ will form. There is also a small O-rich, Co-poor region where $\mathrm{PtO}_{2}$ is the most stable. These limits are shown in Fig. 16. In general, we see that $\mathrm{CoO}_{2}$ limits stability of the delefossites to fairly $\mathrm{Pd} / \mathrm{Pt}$-rich conditions, which will generally increase the formation energy of $\mathrm{Pd} / \mathrm{Pt}$ vacancies, which are the dominant defects in this system. For calculating formation energies in Sec. VA, we assume metal-rich conditions, corresponding to $\mu_{\mathrm{Pd}}=\mu_{\mathrm{Co}}=0$, giving $\mu_{\mathrm{O}}=$ $-1.67 \mathrm{eV}$ for $\mathrm{PdCoO}_{2}$.

\section{APPENDIX D: INTERSTITIAL DEFECT STRUCTURE}

The most stable interstitial structure that we find in $\mathrm{PdCoO}_{2}$ is for the interstitial atom $\left(\mathrm{Pd}_{i}\right)$ to bond to two $\mathrm{O}$ in the layer above, resulting in significant in-plane displacements of two Pd atoms. The bond lengths between the interstitial and the two oxygen atoms are not equal (1.96 and $2.19 \AA$ ), while one of the nearby Pd is significantly more displaced from its original site than the other. The bond lengths between the $\mathrm{Pd}_{i}$ and the nearest two $\mathrm{Pd}$ atoms are approximately equal $(2.34 \AA)$. We can understand this structure as a balance between the $\mathrm{Pd}_{i}$ attempting to form equilibrium length bonds with the two $\mathrm{O}$ atoms above and the two Pd atoms below.

\section{APPENDIX E: STABILITY OF DEFECTS}

To check whether the defects added by irradiation are stable after the initial warm-up to room temperature, we measured the room-temperature resistivity of an irradiated $\mathrm{PtCoO}_{2}$ sample continuously over the course of 20 days. As shown in Fig. 17(a), no change of resistivity was observed during this time, indicating that the defects which remain in the sample after the initial warm-up to room temperature are indeed stable. Consistent with this, we observed no difference in the measurements of the temperature-dependent resistivity taken several months apart. 

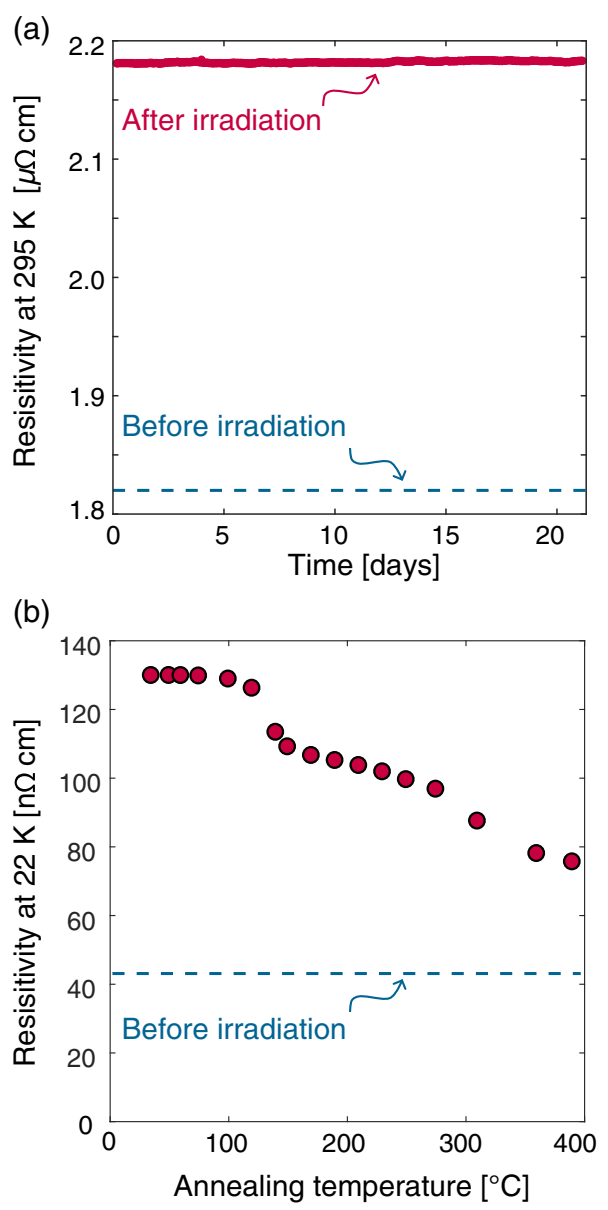

FIG. 17. (a) The room-temperature resistivity of an irradiated $\mathrm{PtCoO}_{2}$ sample measured over the course of 20 days. (b) The resistivity of an irradiated $\mathrm{PtCoO}_{2}$ sample measured at $22 \mathrm{~K}$ as a function of the annealing temperature, at which the sample was kept for $30 \mathrm{~min}$.

At higher temperatures, however, the defects become mobile. To investigate this we have warmed up an irradiated $\mathrm{PtCoO}_{2}$ sample to a series of annealing temperatures between $35^{\circ} \mathrm{C}$ and $390{ }^{\circ} \mathrm{C}$, and kept the sample at each of them for $30 \mathrm{~min}$. After each annealing step, the temperature-dependent resistivity was measured. In Fig. 17(b) we show the resisitivity at $22 \mathrm{~K}$ as a function of annealing temperature. Two clear steps can be seen, indicating two characteristic energies for migration of different types of defects. The fact that the added resistivity is decreased by $35 \%$ after warming up to room temperature indicates that one or more such steps also occur between $22 \mathrm{~K}$ and room temperature.

[1] G. C. Gardner, S. Fallahi, J. D. Watson, and M. J. Manfra, Modified MBE Hardware and Techniques and Role of Gallium Purity for Attainment of Two Dimensional Electron
Gas Mobility > $35 \times 10^{6} \mathrm{~cm}^{2} / \mathrm{Vs}$ in AlGaAs/GaAs Quantum Wells Grown by MBE, J. Cryst. Growth 441, 71 (2016).

[2] L. Banszerus, M. Schmitz, S. Engels, M. Goldsche, K. Watanabe, T. Taniguchi, B. Beschoten, and C. Stampfer, Ballistic Transport Exceeding $28 \mu \mathrm{m}$ in CVD Grown Graphene, Nano Lett. 16, 1387 (2016).

[3] M. Z. Hasan and C. L. Kane, Topological Insulators, Rev. Mod. Phys. 82, 3045 (2010).

[4] X.-L. Qi and S.-C. Zhang, Topological Insulators and Superconductors, Rev. Mod. Phys. 83, 1057 (2011).

[5] N. P. Armitage, E. J. Mele, and A. Vishwanath, Weyl and Dirac Semimetals in Three-Dimensional Solids, Rev. Mod. Phys. 90, 015001 (2018).

[6] T. Liang, Q. Gibson, M. N. Ali, M. Liu, R. J. Cava, and N. P. Ong, Ultrahigh Mobility and Giant Magnetoresistance in the Dirac Semimetal $\mathrm{Cd}_{3} \mathrm{As}_{2}$, Nat. Mater. 14, 280 (2015).

[7] C. Shekhar, A. K. Nayak, Y. Sun, M. Schmidt, M. Nicklas, I. Leermakers, U. Zeitler, Y. Skourski, J. Wosnitza, Z. Liu, Y. Chen, W. Schnelle, H. Borrmann, Y. Grin, C. Felser, and B. Yan, Extremely Large Magnetoresistance and Ultrahigh Mobility in the Topological Weyl Semimetal Candidate NbP, Nat. Phys. 11, 645 (2015).

[8] N. Kumar, Y. Sun, N. Xu, K. Manna, M. Yao, V. Süss, I. Leermakers, O. Young, T. Förster, M. Schmidt, H. Borrmann, B. Yan, U. Zeitler, M. Shi, C. Felser, and C. Shekhar, Extremely High Magnetoresistance and Conductivity in the Type-II Weyl Semimetals $\mathrm{WP}_{2}$ and $\mathrm{MoP}_{2}$, Nat. Commun. 8, 1 (2017).

[9] C. Zhang, Z. Ni, J. Zhang, X. Yuan, Y. Liu, Y. Zou, Z. Liao, Y. Du, A. Narayan, H. Zhang, T. Gu, X. Zhu, L. Pi, S. Sanvito, X. Han, J. Zou, Y. Shi, X. Wan, S. Y. Savrasov, and F. Xiu, Ultrahigh Conductivity in Weyl Semimetal NbAs Nanobelts, Nat. Mater. 18, 482 (2019).

[10] R. D. Shannon, C. T. Prewitt, and D. B. Rogers, Chemistry of Noble Metal Oxides. II. Crystal Structures of Platinum Cobalt Dioxide, Palladium Cobalt Dioxide, Copper Iron Dioxide, and Silver Iron Dioxide, Inorg. Chem. 10, 719 (1971).

[11] A. P. Mackenzie, The Properties of Ultrapure Delafossite Metals, Rep. Prog. Phys. 80, 032501 (2017).

[12] H. Takatsu, S. Yonezawa, S. Mouri, S. Nakatsuji, K. Tanaka, and Y. Maeno, Roles of High-Frequency Optical Phonons in the Physical Properties of the Conductive Delafossite $\mathrm{PdCoO}_{2}$, J. Phys. Soc. Jpn. 76, 104701 (2007).

[13] H. Takatsu, S. Yonezawa, C. Michioka, K. Yoshimura, and Y. Maeno, Anisotropy in the Magnetization and Resistivity of the Metallic Triangular-Lattice Magnet $\mathrm{PdCrO}_{2}$, J. Phys. Conf. Ser. 200, 012198 (2010).

[14] P. Kushwaha, V. Sunko, P. J. W. Moll, L. Bawden, J. M. Riley, N. Nandi, H. Rosner, M. P. Schmidt, F. Arnold, E. Hassinger, T. K. Kim, M. Hoesch, A. P. Mackenzie, and P. D. C. King, Nearly Free Electrons in a $5 d$ Delafossite Oxide Metal, Sci. Adv. 1, e1500692 (2015).

[15] C. W. Hicks, A. S. Gibbs, A. P. Mackenzie, H. Takatsu, Y. Maeno, and E. A. Yelland, Quantum Oscillations and High Carrier Mobility in the Delafossite $\mathrm{PdCoO}_{2}$, Phys. Rev. Lett. 109, 116401 (2012).

[16] H. Takatsu, J. J. Ishikawa, S. Yonezawa, H. Yoshino, T. Shishidou, T. Oguchi, K. Murata, and Y. Maeno, Extremely 
Large Magnetoresistance in the Nonmagnetic Metal $\mathrm{PdCoO}_{2}$, Phys. Rev. Lett. 111, 056601 (2013).

[17] R. Daou, R. Frésard, S. Hébert, and A. Maignan, Large Anisotropic Thermal Conductivity of the Intrinsically TwoDimensional Metallic Oxide $\mathrm{PdCoO}_{2}$, Phys. Rev. B 91, 041113(R) (2015).

[18] P. J. W. Moll, P. Kushwaha, N. Nandi, B. Schmidt, and A. P. Mackenzie, Evidence for Hydrodynamic Electron Flow in $\mathrm{PdCoO}_{2}$, Science 351, 1061 (2016).

[19] N. Kikugawa, P. Goswami, A. Kiswandhi, E. S. Choi, D. Graf, R. E. Baumbach, J. S. Brooks, K. Sugii, Y. Iida, M. Nishio, S. Uji, T. Terashima, P. M. C. Rourke, N. E. Hussey, H. Takatsu, S. Yonezawa, Y. Maeno, and L. Balicas, Interplanar Coupling-Dependent Magnetoresistivity in High-Purity Layered Metals, Nat. Commun. 7, 10903 (2016).

[20] N. Nandi, T. Scaffidi, P. Kushwaha, S. Khim, M. E. Barber, V. Sunko, F. Mazzola, P. D. C. King, H. Rosner, P. J. W. Moll, M. König, J.E. Moore, S. Hartnoll, and A.P. Mackenzie, Unconventional Magneto-Transport in Ultrapure $\mathrm{PdCoO}_{2}$ and $\mathrm{PtCoO}_{2}$, npj Quantum Mater. 3, 66 (2018).

[21] C. Putzke, M. D. Bachmann, P. McGuinness, E. Zhakina, T. Oka, R. Moessner, M. König, S. Khim, A. P. Mackenzie, and P. J. W. Moll, h/e Oscillations in Interlayer Transport of Delafossites, arXiv:1902.07331.

[22] H. Usui, M. Ochi, S. Kitamura, T. Oka, D. Ogura, H. Rosner, M. W. Haverkort, V. Sunko, P. D. C. King, A. P. Mackenzie, and K. Kuroki, Hidden Kagome-Lattice Picture and Origin of High Conductivity in Delafossite $\mathrm{PtCoO}_{2}$, Phys. Rev. Mater. 3, 045002 (2019).

[23] P. Kushwaha, H. Borrmann, S. Khim, H. Rosner, P. J. W. Moll, D. A. Sokolov, V. Sunko, Y. Grin, and A. P. Mackenzie, Single Crystal Growth, Structure, and Electronic Properties of Metallic Delafossite $\mathrm{PdRhO}_{2}$, Cryst. Growth Des. 17, 4144 (2017).

[24] M. Berger, J. Coursey, M. Zucker, and J. Chang, STAR, PSTAR, and ASTAR: Computer Programs for Calculating Stopping-Power and Range Tables for Electrons, Protons, and Helium Ions (version 1.2.3), http://physics .nist.gov/Star, https://physics.nist.gov/PhysRefData/Star/ Text/ESTAR.html.

[25] P. J. Moll, Focused Ion Beam Microstructuring of Quantum Matter, Annu. Rev. Condens. Matter Phys. 9, 147 (2018).

[26] G. W. Iseler, H. I. Dawson, A. S. Mehner, and J. W. Kauffman, Production Rates of Electrical Resistivity in Copper and Aluminum Induced by Electron Irradiation, Phys. Rev. 146, 468 (1966).

[27] A. Legris, F. Rullier-Albenque, E. Radeva, and P. Lejay, Effects of Electron Irradiation on $\mathrm{YBa}_{2} \mathrm{Cu}_{3} \mathrm{O}_{7-\delta}$ Superconductor, J. Phys. I (France) 3, 1605 (1993).

[28] J. C. Meyer, F. Eder, S. Kurasch, V. Skakalova, J. Kotakoski, H. J. Park, S. Roth, A. Chuvilin, S. Eyhusen, G. Benner, A. V. Krasheninnikov, and U. Kaiser, Accurate Measurement of Electron Beam Induced Displacement Cross Sections for Single-Layer Graphene, Phys. Rev. Lett. 108, 196102 (2012).

[29] N. F. Mott, The Scattering of Fast Electrons by Atomic Nuclei, Proc. R. Soc. A 124, 425 (1929).

[30] N. F. Mott, The Polarisation of Electrons by Double Scattering, Proc. R. Soc. A 135, 429 (1932).
[31] W. A. McKinley and H. Feshbach, The Coulomb Scattering of Relativistic Electrons by Nuclei, Phys. Rev. 74, 1759 (1948).

[32] T. Lijian, H. Qing, and L. Zhengming, Analytic Fitting to the Mott Cross Section of Electrons, Radiat. Phys. Chem. 45, 235 (1995).

[33] M. J. Boschini, C. Consolandi, M. Gervasi, S. Giani, D. Grandi, V. Ivanchenko, P. Nieminem, S. Pensotti, P. G. Rancoita, and M. Tacconi, An Expression for the Mott Cross Section of Electrons and Positrons on Nuclei with Z Up to 118, Radiat. Phys. Chem. 90, 39 (2013).

[34] $\beta=\sqrt{1-\left[m_{e} c^{2} /\left(m_{e} c^{2}+E_{K}\right)\right]^{2}}$, where $m_{e}$ is the electron rest mass.

[35] The scattering angle $\vartheta$ and the transferred energy $E$ are related by $E=E_{\max } \sin ^{2} \vartheta / 2 . E_{\max }$ is the maximum energy that can be transferred, $E_{\max }=2 E_{K}\left(E_{K}+2 m_{e} c^{2}\right) / M c^{2}$. $E_{K}$ is the kinetic energy of the incoming electron, and $m_{e}$ and $M$ are the masses of the electron and the nucleus, respectively.

[36] Technically, the accelerator energy can be reduced down to $0.3 \mathrm{MeV}$; however, this requires a stabilization time of a few days, and was therefore not feasible in the limited beam time.

[37] N. Kikugawa and Y. Maeno, Non-Fermi-Liquid Behavior in $\mathrm{Sr}_{2} \mathrm{RuO}_{4}$ with Nonmagnetic Impurities, Phys. Rev. Lett. 89, 117001 (2002).

[38] Y. Fukuzumi, K. Mizuhashi, K. Takenaka, and S. Uchida, Universal Superconductor-Insulator Transition and $T_{c}$ Depression in Zn-Substituted High- $T_{c}$ Cuprates in the Underdoped Regime, Phys. Rev. Lett. 76, 684 (1996).

[39] F. Rullier-Albenque, P. A. Vieillefond, H. Alloul, A. W. Tyler, P. Lejay, and J. F. Marucco, Universal $T_{c}$ Depression by Irradiation Defects in Underdoped and Overdoped Cuprates?, Europhys. Lett. 50, 81 (2000).

[40] B. Grabowski, L. Ismer, T. Hickel, and J. Neugebauer, Ab Initio Up to the Melting Point: Anharmonicity and Vacancies in Aluminum, Phys. Rev. B 79, 134106 (2009).

[41] C. Freysoldt, B. Grabowski, T. Hickel, J. Neugebauer, G. Kresse, A. Janotti, and C. G. Van de Walle, First-Principles Calculations for Point Defects in Solids, Rev. Mod. Phys. 86, 253 (2014).

[42] S. M. Foiles, M. I. Baskes, and M. S. Daw, EmbeddedAtom-Method Dunctions for the fcc Metals $\mathrm{Cu}, \mathrm{Ag}, \mathrm{Au}, \mathrm{Ni}$, Pd, Pt, and Their Alloys, Phys. Rev. B 33, 7983 (1986).

[43] T. R. Mattsson and A. E. Mattsson, Calculating the Vacancy Formation Energy in Metals: Pt, Pd, and Mo, Phys. Rev. B 66, 214110 (2002).

[44] R. Nazarov, T. Hickel, and J. Neugebauer, Vacancy Formation Energies in fcc Metals: Influence of Exchange-Correlation Functionals and Correction Schemes, Phys. Rev. B 85, 144118 (2012).

[45] H.-E. Schaefer, Investigation of Thermal Equilibrium Vacancies in Metals by Positron Annihilation, Phys. Status Solidi A 102, 47 (1987).

[46] H. Ullmaier, P. Ehrhart, P. Jung, and H. Schultz, Atomic Defects in Metals (Springer, New York, 1991), Vol. 3.

[47] J. M. Ziman, Principles of the Theory of Solids (Cambridge University Press, Cambridge, England, 1972).

[48] J. M. Harris, Y. F. Yan, P. Matl, N. P. Ong, P. W. Anderson, T. Kimura, and K. Kitazawa, Violation of Kohler's Rule in 
the Normal-State Magnetoresistance of $\mathrm{YBa}_{2} \mathrm{Cu}_{3} \mathrm{O}_{7-\delta}$ and $\mathrm{La}_{2} \mathrm{Sr}_{\mathrm{x}} \mathrm{CuO}_{4}$, Phys. Rev. Lett. 75, 1391 (1995).

[49] C. W. Groth, M. Wimmer, A. R. Akhmerov, and X. Waintal, Kwant: A Software Package for Quantum Transport, New J. Phys. 16, 063065 (2014).

[50] C. A. C. Garcia, J. Coulter, and P. Narang, Optoelectronic Response of Type-I Weyl Semimetals TaAs and NbAs from First Principles, Phys. Rev. Research 2, 013073 (2020).

[51] P. T. Coleridge, Small-Angle Scattering in TwoDimensional Electron Gases, Phys. Rev. B 44, 3793 (1991).

[52] Research data supporting this publication can be accessed at https://doi.org/10.17630/3d6fde13-8bfe-491f8233-32c5f4db6c5e.
[53] J. Sólyom, Fundamentals of the Physics of Solids: Volume II: Electronic Properties (Springer, Berlin, 2008).

[54] I. R. Lapidus, Quantum-Mechanical Scattering in Two Dimensions, Am. J. Phys. 50, 45 (1982).

[55] J. P. Perdew, K. Burke, and M. Ernzerhof, Generalized Gradient Approximation Made Simple, Phys. Rev. Lett. 77, 3865 (1996).

[56] G. Kresse and J. Furthmüller, Efficient Iterative Schemes for Ab Initio Total-Energy Calculations Using a Plane-Wave Basis Set, Phys. Rev. B 54, 11169 (1996).

[57] K. P. Ong, J. Zhang, J. S. Tse, and P. Wu, Origin of Anisotropy and Metallic Behavior in Delafossite $\mathrm{PdCoO}_{2}$, Phys. Rev. B 81, 115120 (2010). 